\title{
Nonlinear resonance and synchronization in the ring of unidirectionally coupled Toda oscillators
}

\author{
Anton Dvorak ${ }^{1, \mathrm{a}}$, Vladimir Astakhov ${ }^{1}$, Przemyslaw Perlikowski ${ }^{2, \mathrm{~b}}$, \\ and Tomasz Kapitaniak ${ }^{2, c}$ \\ 1 Department of Radioelectronics and Telecommunications, Yuri Gagarin State Technical \\ University of Saratov, Polytechnicheskaya 77, Saratov 410054, Russia \\ 2 Division of Dynamics, Lodz University of Technology, Stefanowskiego 1/15, 90-924 Lodz, \\ Poland
}

Received 31 January 2016 / Received in final form 29 July 2016

Published online 22 November 2016

\begin{abstract}
In the ring of unidirectionally coupled Toda oscillators the nonlinear resonance and the synchronization are investigated. It is shown how the nonlinear resonance affects the structure of the main synchronization region. As a result of nonlinear resonance we observe the coexistence of two stable limit cycles near the resonant frequency, which leads to coexistence of periodic and quasi-periodic regimes within the synchronization region.
\end{abstract}

\section{Introduction}

Oscillator chains can be divided into two groups: self-excited, which demonstrate stable limit cycle without external driving [1-5] and strictly dissipative chains, which have stable steady state without forcing [6-9]. For the ring of unidirectionally coupled Duffing oscillators [2] and for the ring of unidirectionally coupled Toda oscillators with nonlinear coupling function [3], it has been shown that with the increase of coupling coefficient the stable fixed point undergoes Andronov-Hopf bifurcation where the stable limit cycle may appear $[3,4]$. Hence, the system can reach a stable fixed point (when the coupling coefficient of the system is small) or stable limit cycle (when the coupling coefficient is above the threshold of Andronov-Hopf bifurcation). The systems of coupled Toda and Duffing oscillators demonstrate the effect of nonlinear resonance [6-11]. Simulationousely as for all self-oscillatory coupled systems we expect the appearence of synchronization [12-15]. It has been shown, that in the case of strong nonlinearity the main synchronization region of self-oscillatory systems can have some peculiarities, such as asymmetry and stretched form of synchronization tongue $[16,17]$. Neimark-Sacker curves for such systems do not meet saddle-node curves of limit cycles at its cusp points. It is interesting to see, how the nonlinear

\footnotetext{
a e-mail: dvorakaa@sstu.ru

b e-mail: przemyslaw.perlikowski@p.lodz.pl

c e-mail: tomaszka@p.lodz.pl
} 

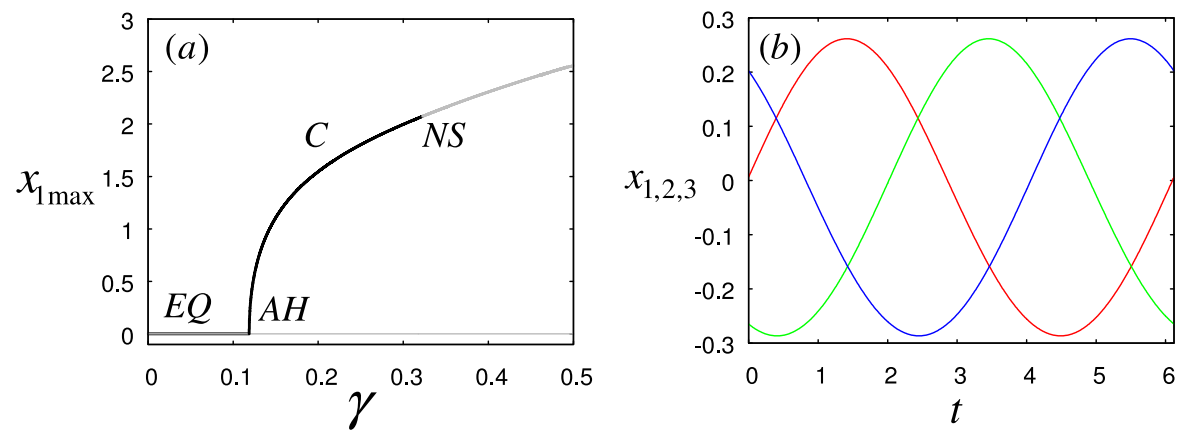

Fig. 1. Bifurcation diagram of steady state and limit cycle for the system (1) without external force $(A=0)$ (a) where AH is the point of Andronov-Hopf bifurcation, NS is the point of Neimark-Sacker bifurcation; variables of partial oscillators $x_{1}, x_{2}, x_{3}$ versus time at $\gamma=0.12$ without external force $(F=0)(b)$.

resonance affects the synchronization in the system and the peculiarities of the structure of the main synchronization region.

In this paper we consider unidirectionally coupled Toda oscillators with ring topology of coupling. It has been shown, that increasing the coupling strength leads to selfoscillation birth $[2,3]$. Hence, the system which is investigated in our paper, represents a special type of generator under external force. The most interesting effect which takes place in the forced generators is synchronization. We show that the main synchronization region has classical structure only for small amplitude of driving force. For larger values of amplitude, we observe ranges where different solutions coexist, hence it is non-classical behavior. To explain this non-typical structure, we study the resonance in the system under the threshold of self-oscillation birth.

\section{The model}

We consider the ring of three unidirectionally coupled Toda oscillators with the harmonic force acting on one of them:

$$
\begin{aligned}
& \dot{x}_{1}=y_{1}, \\
& \dot{y}_{1}=1-\exp \left(x_{1}\right)-\alpha y_{1}+\gamma H\left(x_{3}\right)+F \sin (\omega t), \\
& \dot{x}_{2}=y_{2}, \\
& \dot{y}_{2}=1-\exp \left(x_{2}\right)-\alpha y_{2}+\gamma H\left(x_{1}\right), \\
& \dot{x}_{3}=y_{3} \\
& \dot{y}_{3}=1-\exp \left(x_{3}\right)-\alpha y_{3}+\gamma H\left(x_{2}\right),
\end{aligned}
$$

where $x_{1,2,3}, y_{1,2,3}$, are the dynamical variables of the system, $\alpha$ is the damping coefficient, $\gamma$ is the coupling coefficient $(\gamma>0)$ and $H(x)=\exp (x)-1$ is the coupling function.

The investigations of the system have been conducted using the XppAut package [18]. Limit cycle branches and bifurcations of limit cycles have been obtained by Auto07p [19] software, time series and projections of phase portraits have been obtained by numerical integration of differential equations using Runge-Kutta method.

The system without external force $(F=0)$ with $\alpha=0.1$ has the stable steady state for the coupling parameter $\gamma<0.1189$, Andronov-Hopf bifurcation occurs at $\gamma=$ 0.1189 and the stable limit cycle is born. Bifurcation diagram is shown in Fig. 1(a). 

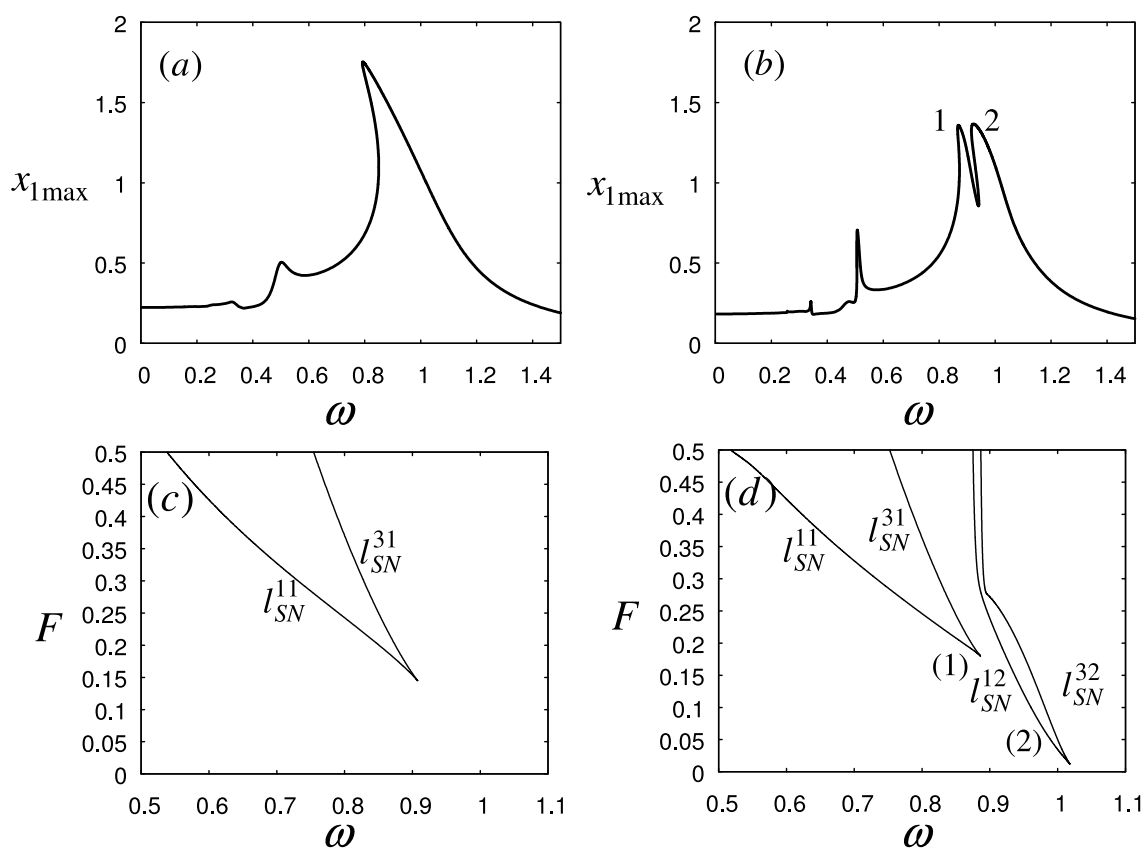

Fig. 2. Resonance curves for the system (1) for $\gamma=0$ and $F=0.25$ (a), $\gamma=0.11$ and $F=0.2$ (b), lines of saddle-node bifurcations of resonance limit cycles for $\gamma=0$ (c), $\gamma=0.11$ (d).

Further increase of coupling coefficient leads to Neimark-Sacker bifurcation of the limit cycle and the appearance of complex dynamics. In all figures we mark the equilibrium as EQ and limit cycles with letter $\mathrm{C}$ using numerical subscripts, if we observe more than one solution.

The periodic regime is characterized by phase shifts between partial oscillators equal to $T / 3$, where $T$ is period of oscillations (see Fig. 1(b)).

\section{Nonlinear resonance in the system}

To observe resonance in the system (1), we choose two values of the coupling coefficient $\gamma$, which correspond to stable steady state in the system without external forcing. The first value fixed as $\gamma=0$ demonstrates the resonance properties of partial oscillator. Bifurcation structure of the driven Toda oscillator on the parameter space of amplitude and frequency of driving force is studied [20]. Nonlinear resonance leads to the appearance of «resonance horns» on the parameter space - areas, which are formed by lines of saddle-node bifurcations. Inside these areas two resonance limit cycles coexist. Figure 2(a) shows the resonance curve of the system with $\gamma=0$, where the resonance 1:1 is clearly seen whereas Fig. 2(c) shows the bottom part of resonance horn 1:1.

Figure 2(b) shows the resonance curve when $\gamma=0.11$. Here, we can see two resonances, which are signed by 1 and 2 . The corresponding two-parameter diagram is shown in Fig. 2(b). Here we can see two resonance horns for these two resonances labelled by 1 and 2 .

Time series of $x_{1}(t), x_{2}(t)$ and $x_{3}(t)$ for coexisting resonance limit cycles for the first and the second resonances are shown in Figs. 3(a, b) and 3(c, d) respectively. Let us call first resonance as partial oscillator resonance and the second resonance as ring 

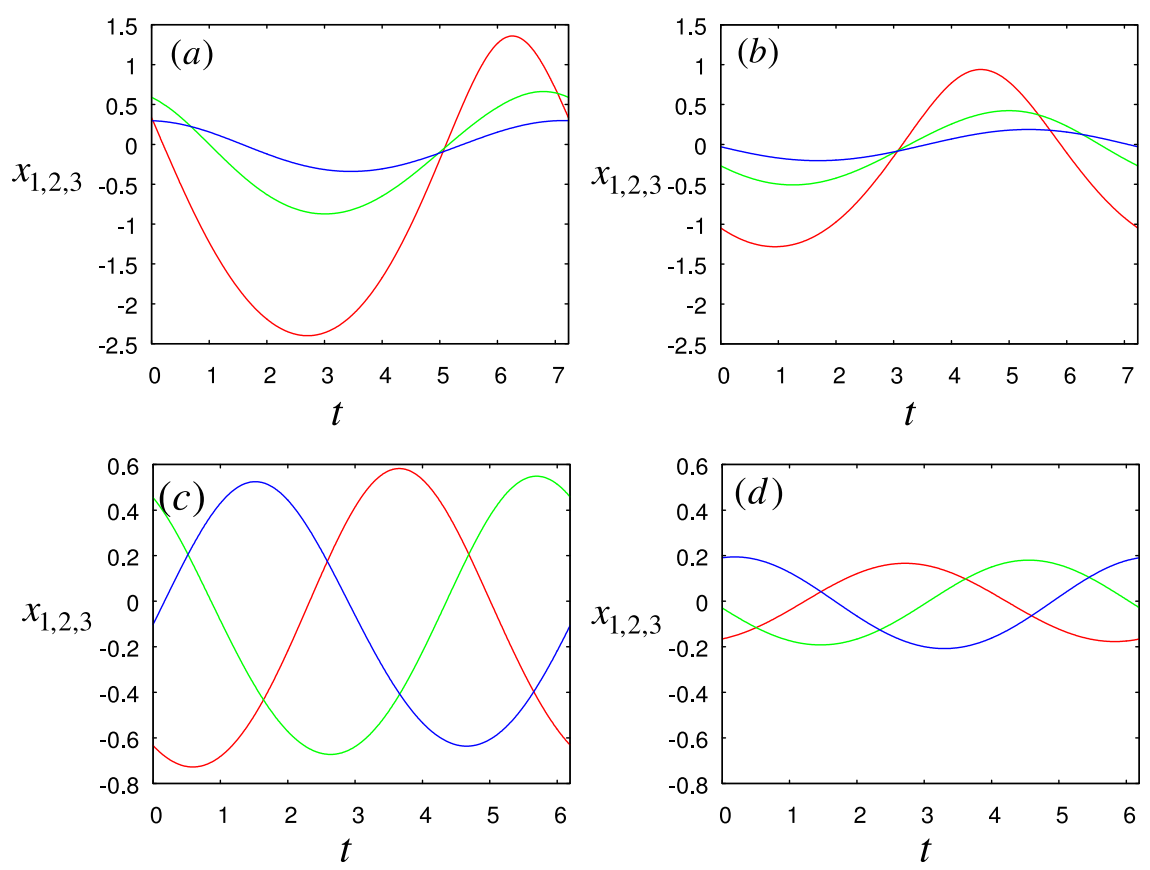

Fig. 3. Variables of partial oscillators $x_{1}, x_{2}, x_{3}$ versus time for the coexisting limit cycles of the system (1) for partial oscillator resonance (a, b) $\gamma=0.11, \omega=0.869, F=0.02$ and ring resonance $(\mathrm{c}, \mathrm{d}) \gamma=0.11, \omega=1.0135, F=0.015$.

resonance. We can see, that phase shifts between oscillations for the first resonance are near zero, and for the second resonance oscillations are similar to the self-oscillations in the autonomous system (Fig. 1): the amplitudes of partial oscillators are different, but phase shifts are $T / 3$, where $T$ is the period of the driving force.

We can see, that the coupling in the system leads to the appearance of the new resonance, determined by the ring structure of the coupled oscillators.

\section{The structure of the main synchronization region}

In order to observe synchronization in the system we fix the internal parameter values as $\alpha=0.1, \gamma=0.12$ (which corresponds to the stable limit cycle in an autonomous system) and investigate the main synchronization region on the plane $(\omega, A)$ of the external force parameters.

The results of the detailed investigations of dynamical regimes and transitions between them are shown in Fig. 4 . In region $\mathrm{A}$, there are three limit cycles: stable $C_{1}$, saddle $C_{2}$ and $C_{3}$ (Fig. 5(a)). On the lines $l_{S N}^{1}$ and $l_{S N}^{1}$ there are saddle-node bifurcations of cycles $C_{1}$ and $C_{2}$ on torus (Fig. 6(a) shows the bifurcation). On the line $l_{S N}^{3}$ there is saddle-saddle bifurcation of cycles $C_{2}$ and $C_{3}$ (Fig. 6(b)), so in the region $\mathrm{B}$ only one cycle $C_{1}$ is left (Fig. $5(\mathrm{~b})$ ). In region $\mathrm{C}$ between the lines $l_{S N}^{1^{\prime}}$ and $l_{T D}$ there are hysteretic transitions between the limit cycle and the torus, in this region the stable torus and the limit cycle coexist (Fig. $5(\mathrm{c})$ ). On line $l_{T D}$ the coexisting torus disappears. On line $l_{N S}^{3}$ the torus converges to cycle $C_{3}$, which becomes stable and in region D two stable cycles $C_{1}$ and $C_{3}$ coexist (Fig. 5(d)). On line $l_{S N}^{3^{\prime}}$ there is saddlenode bifurcation of cycles $C_{2}$ and $C_{3}$, so in region B still only one limit cycle $C_{1}$ is left. Lines $l_{S N}^{3}, l_{S N}^{3^{\prime}}, l_{N S}^{3}$ and $l_{T D}$ are connected in the point $p_{3}$. The bifurcation diagram 


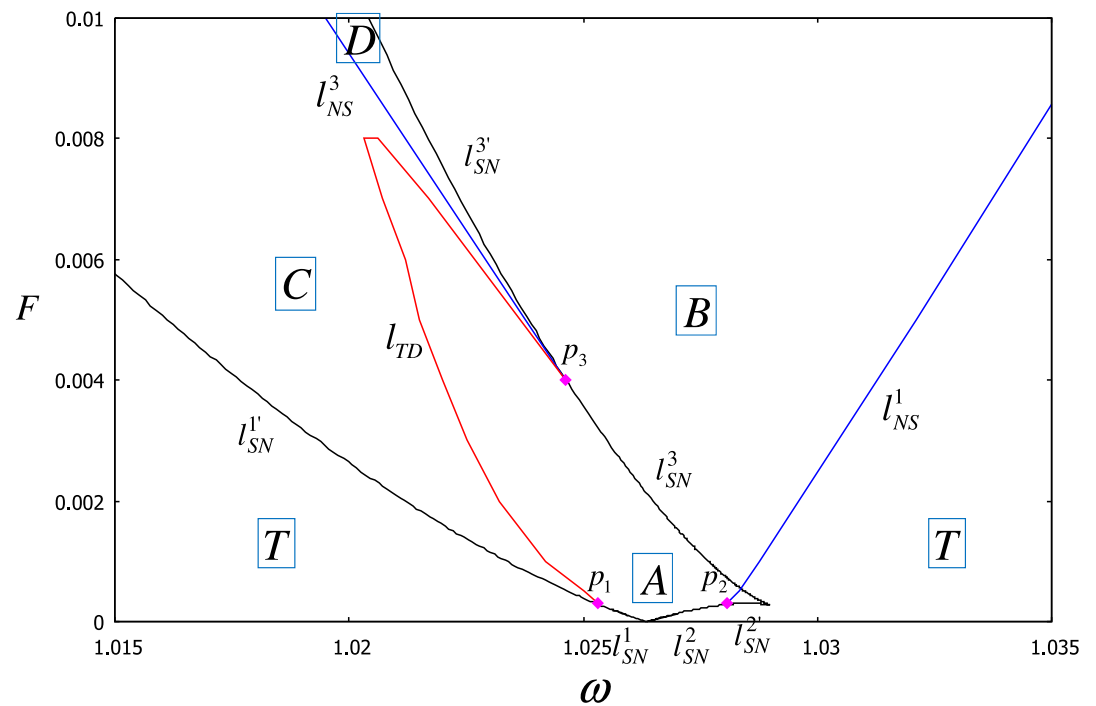

Fig. 4. Bifurcation lines of the system (1) in the synchronization region 1:1. Parameter values are $\alpha=0.1, \gamma=0.12$. $l_{S N}$ are lines of saddle-node bifurcations of limit cycles, $l_{N S}$ are lines of supercritical Neimark-Sacker bifurcations, $l_{T D}$ is the line of torus disappear, which is diagnosed by following phase trajectory of the system.
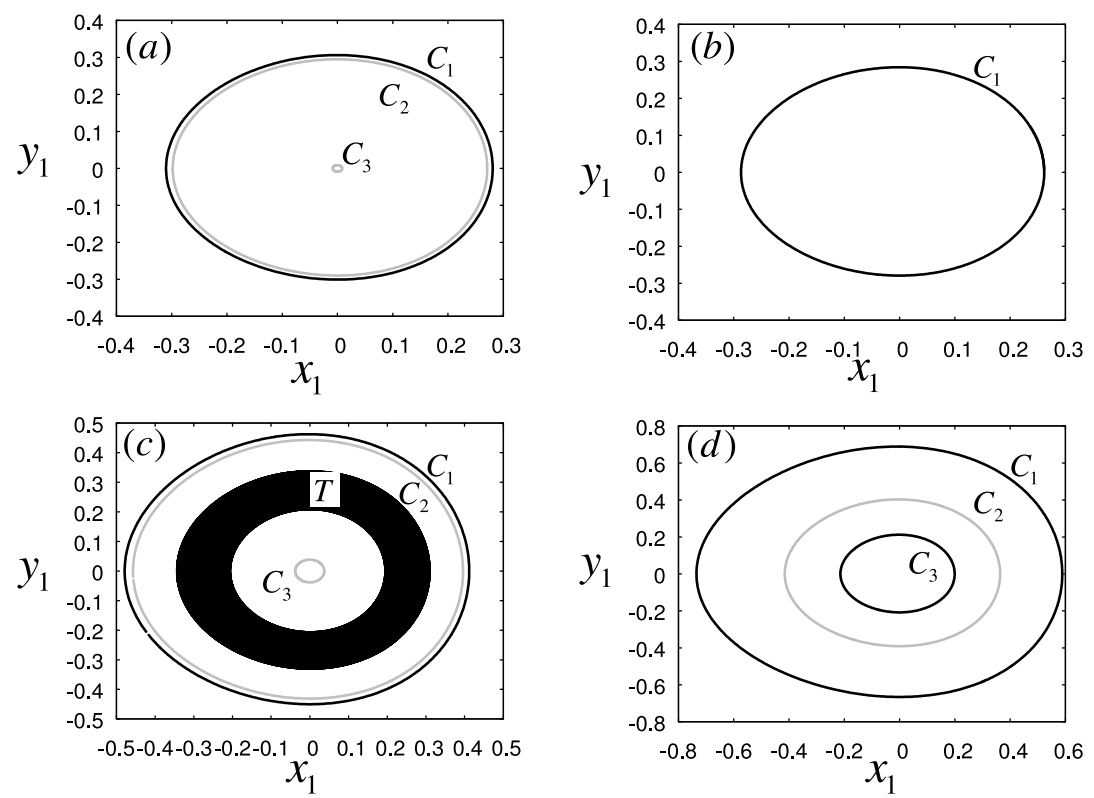

Fig. 5. Characteristic phase portrait projections for stable (black) and unstable (gray) solutions of system (1) for $\alpha=0.1, \gamma=0.12$ (a) for area $A$, (b) for area $B$, (c) for area $C$, (d) for the $D$ of the parameter plane $(\omega, F)$ in the Fig. 4. Phase portrait projections of coexisting regimes have been obtained by numerical solution with different initial values of variables. 

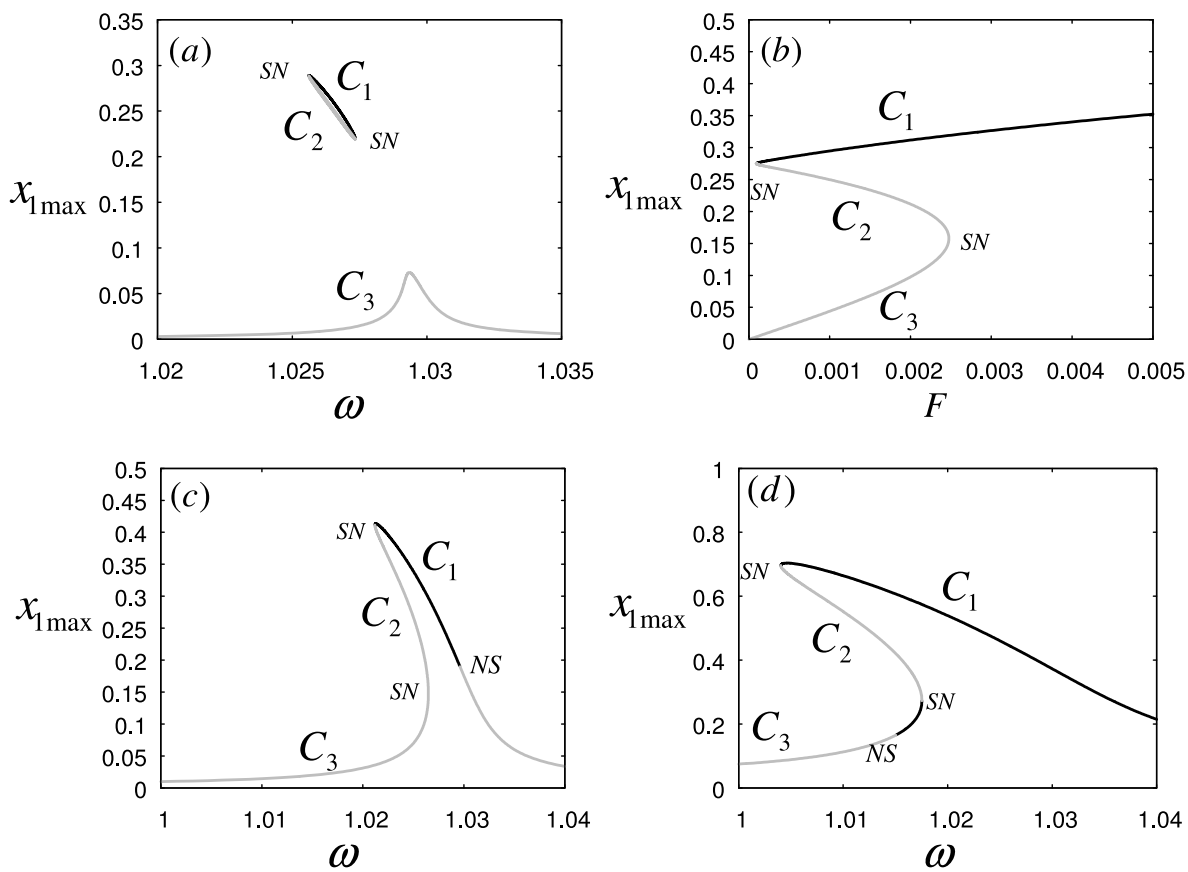

Fig. 6. Bifurcation diagrams for stable (black) and unstable (gray) limit cycles of system (1) for $\alpha=0.1, \gamma=0.12$ (a) for $F=0.00025$, (b) for $\omega=1.026$, (c) for $F=0.002$, (d) for $F=0.01$. SN are points of saddle-node bifurcations, NS are points of NeimarkSacker bifurcations.

for the case when we are under this point is shown in Fig. 6(c), when we are above this point - in Fig. 6(d). On line $l_{N S}^{1}$ there is Neimark-Sacker bifurcation of cycle $C_{1}$.

Summarizing, in regions A and B we see only one stable limit cycle in the phase space of the system. In region $\mathrm{C}$ we see the coexisting limit cycle and the torus. In region $\mathrm{D}$ we observe two coexisting limit cycles. The regions where only the torus is stable are marked by $\mathrm{T}$.

Such a structure of the synchronization area is closely connected with the appearance of the new resonance. Figure 7 shows the bifurcation lines of the system under the threshold of self-oscillation birth (a) and over it (b). Under the threshold of self-oscillation birth we can see saddle-node bifurcation lines of resonance limit cycles, which form the area of coexisting regimes. Over the threshold of self-oscillation birth in the bottom part of the parameter plane (by small values of external force amplitude) we see classical Arnold tongue formed by saddle-node bifurcation lines on a torus. In the upper part of the parameter plane we see the coexisting resonance cycles, as it was under the threshold of self-oscillation birth but now each of them undergoes Neimark-Sacker bifurcation.

Hence, in the bottom part of the parameter plane we observe the classical case of synchronization. In the upper part we see the effects of the nonlinear resonance in the ring.

In order to see the processes in the system which take place by the transition through the threshold of self-oscillation birth under external force, we fix the driving force amplitude as $F=0.015$ and increase the coupling coefficient. We observe the dynamical regimes of the system on the parameter plane $(\omega, \gamma)$ (Fig. 8). For the weak coupling only one limit cycle is observed (Fig. 9(a), Fig. 8 region B). With the increase of the coupling coefficient the softening characteristic of stiffness is visible, hence we 

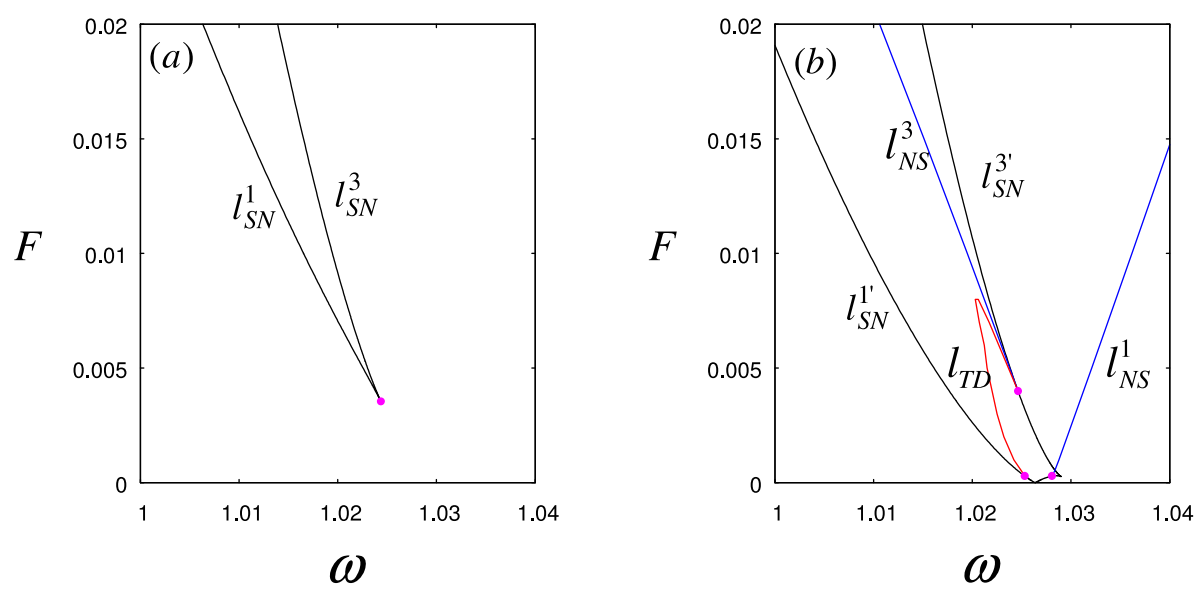

Fig. 7. Bifurcation lines of system (1) for $\gamma=0.11$ (a) and $\gamma=0.12$ (b).

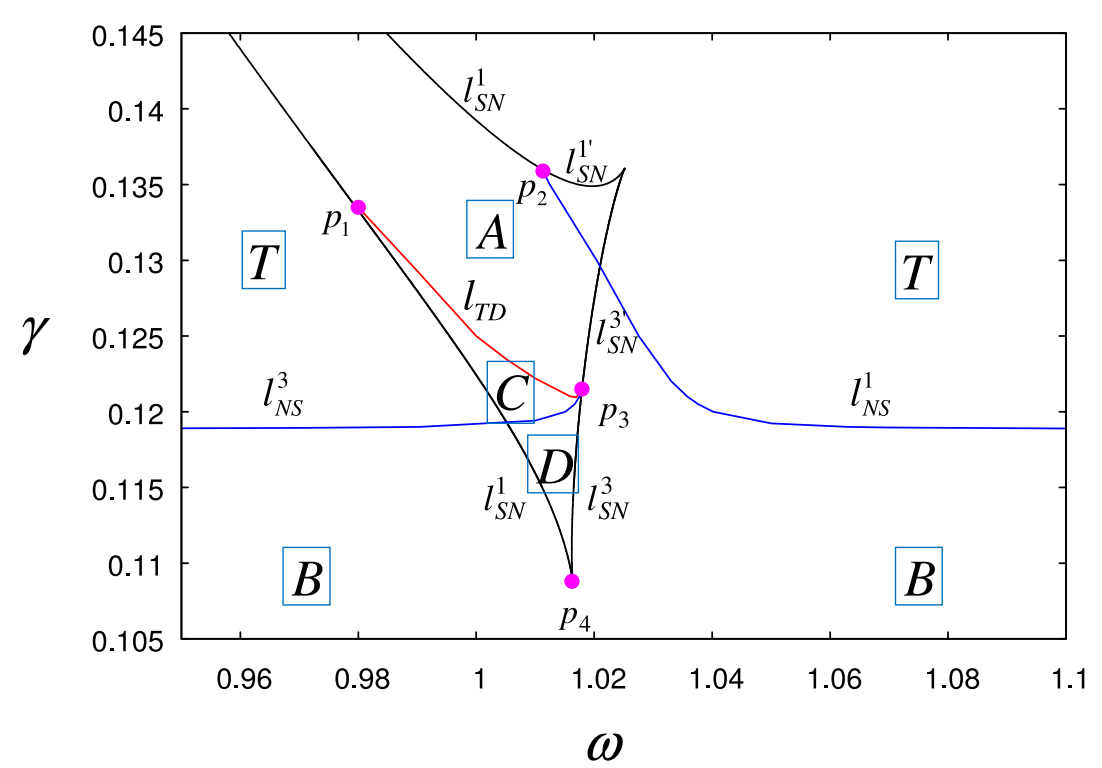

Fig. 8. Bifurcation lines for system (1) on the parameter plane $(\omega, \gamma)$. Other parameter values are $\alpha=0.1$ and $F=0.015 . l_{S N}$ are lines of saddle-node bifurcations of limit cycles, $l_{N S}$ are lines of supercritical Neimark-Sacker bifurcations, $l_{T D}$ is the line where the torus disappears, which is diagnosed by the following phase trajectory of the system.

observe coexistence of the stable limit cycles (Fig. 9(b)). In Fig. 8 between the lines $l_{S N}^{1}$ and $l_{S N}^{3}$ (region D) two stable limit cycles $C_{1}$ and $C_{3}$ and saddle cycle $C_{2}$ are observed. Point $p_{4}$ here is the cusp point of saddle-node bifurcation lines $l_{S N}^{1}$ and $l_{S N}^{3}$.

Over the threshold of self-oscillation birth (which occurs in the system without driving at $\gamma=0.1189$ ) each of the stable limit cycles undergo an Neimark-Sacker bifurcation (Fig. 9(c)) on lines $l_{N S}^{1}$ and $l_{N S}^{3}$ (for cycles $C_{1}$ and $C_{3}$ respectively). Over line $l_{N S}^{3}$ in region $\mathrm{C}$, there are coexisting limit cycle $C_{1}$ and torus (it disappears on line $l_{T D}$ ), so over this line in region A only one stable limit cycle $C_{1}$ is left. Point $p_{3}$ is point where the lines $l_{N S}^{3}$ and $l_{T D}$ meet line $l_{S N}^{3}$, whereas point $p_{1}$ is the point where line $l_{T D}$ meets line $l_{S N}^{1}$. On line $l_{S N}^{3^{\prime}}$ there is saddle-saddle bifurcation of limit cycles 

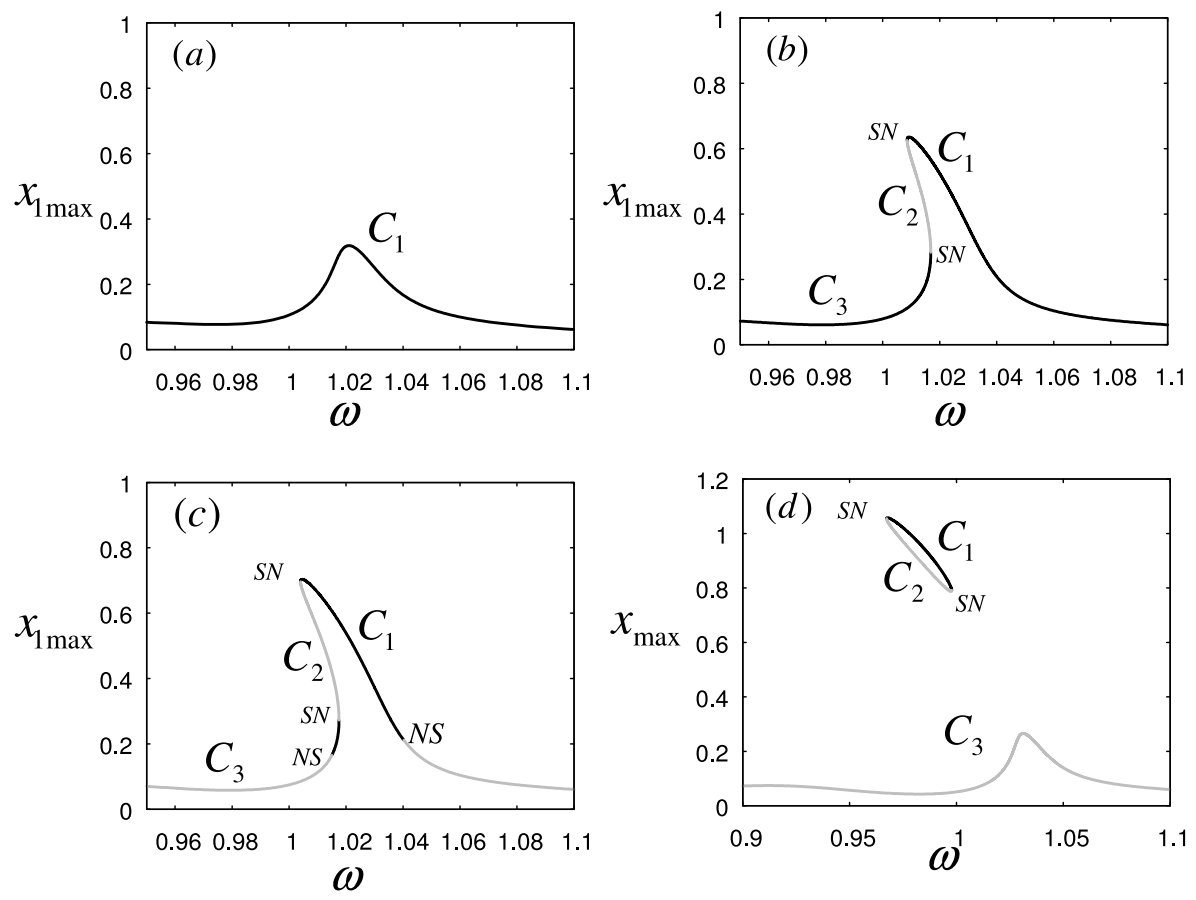

Fig. 9. One-parametric diagrams of limit cycles of system (1) with $\alpha=0.1, F=0.015$ and (a) $\gamma=0.1$, (b) $\gamma=0.117$, (c) $\gamma=0.12$, (d) $\gamma=0.145$. SN are points of saddle-node bifurcations, NS are points of Neimark-Sacker bifurcations.

$C_{2}$ and $C_{3}$. On the upper side of the parameter plane we see another saddle-node bifurcation line $l_{S N}^{1}$ for cycles $C_{1}$ and $C_{2}$. Bifurcation diagram for this case can be seen in the Fig. 9(d). Here, we can observe saddle-node bifurcations of cycles $C_{1}$ and $C_{2}$ on lines $l_{S N}^{1}$ while cycle $C_{3}$ is unstable. Point $p_{2}$ is the point where line $l_{N S}^{1}$ meets line $l_{S N}^{1}$. On line $l_{S N}^{3^{\prime}}$ there is saddle-saddle bifurcation of limit cycles $C_{1}$ and $C_{2}$.

Hence, in region $\mathrm{B}$ we can only see only one stable limit cycle in the phase space of the system, which corresponds to the forced oscillations of the system. In region $D$ we see two coexisting limit cycles, while in region $C$ we observe the coexisting limit cycle and the torus. In region A there is one stable limit cycle. The regions where only the torus is stable are marked by $\mathrm{T}$.

We can see that under the threshold of self-oscillation birth two coexisting stable limit cycles can be observed in the phase space of the system. Over the threshold of self-oscillation birth, the coexisting limit cycles undergo Neimark-Sacker bifurcations, which lead to the coexistence of periodic and quasi-periodic solutions.

\section{Conclusion}

The main conclusion from our paper is that the peculiarities in the structure of synchronization region such as coexisting periodic and quasi-periodic solutions are caused by nonlinear resonance. When the coupling parameter of the system is close to zero, we see only one resonance near the frequency of partial oscillators. Under the threshold of self-oscillation birth, the increase of the coupling strength leads to the appearance of the new resonance, which we call a ring resonance, which is caused by the ring structure of the system (three coupled systems). Nonlinear resonances lead 
to the coexistence of two limit cycles. Over the threshold of self-oscillation birth we see the interaction between external oscillations and the inner oscillatory mode of the system, which leads to the appearance of the synchronization region in the parameter plane. The bottom part of this synchronization region has the classic form of Arnold tongue, while the upper part of it is the result of nonlinear resonance (coexistence of different attractors).

A.D. thanks the Lodz University of Technology for hospitality. V.A. acknowledges the support of Ministry of Education and Science of Russian Federation (state task N. 1694). P.P. acknowledges support by Lodz University of Technology own Scholarship Fund. We thank Dr Marcin Kapitaniak for useful comments.

\section{References}

1. W. Ebeling, P.S. Landa, V.G. Ushakov, Phys. Rev. E 63, 046601 (2001)

2. P. Perlikowski, S. Yanchuk, M. Wolfrum, A. Stefanski, P. Mosiolek, T. Kapitaniak, Chaos 20, 03111 (2010)

3. A. Dvorak, P. Kuzma, P. Perlikowski, V. Astakhov, T. Kapitaniak, Eur. Phys. J. Special Topics 222, 2429 (2013)

4. A.A. Dvorak, V.V. Astakhov, N.V. Stankevich, Dinamika kolcevogo generatora iz odnonapravlenno sviazannyh oscillatorov Tody. "Trudy shkoly-seminara" Volny-2013 Fizicheskii Fakultet MGU (2013) (in Russian)

5. K. Czolczynski, P. Perlikowski, A. Stefanski, T. Kapitaniak, Chaos Solitons Fractals 32, $937(2007)$

6. K. Geist, W. Lauterborn, Physica D 31, 103 (1988)

7. K. Geist, W. Lauterborn, Physica D 41, 1 (1990)

8. K. Geist, W. Lauterborn, Physica D 52, 551 (1991)

9. J. Kozlowski, U. Parlitz, W. Lauterborn, Phys. Rev. E. 51, 1861 (1995)

10. S.I. Arroyo, D.H. Zanette, Eur. Phys. J. B 89, 12 (2016)

11. G. Habib, T. Detroux, R. Viguie, G. Kerschen, Mech. Syst. Signal Proc. 52, 17 (2015)

12. A. Balanov, N. Janson, D. Postnov, O. Sosnovtseva, Synchronization: From Simple to Complex (Springer Science \& Business Media, 2008)

13. A. Pikovsky, Y.L. Maistrenko, Synchronization: Theory and Application (Springer Science \& Business Media, 2012)

14. G.V. Osipov, J. Kurths, Ch. Zhou, Synchronization in Oscillatory Networks (Springer Science \& Business Media, 2007)

15. S. Yanchuk, A. Stefanski, T. Kapitaniak, J. Wojewoda, Phys. Rev. E 73, 016209 (2006)

16. R. Mettin, U. Parlitz, W. Lauterborn, Int. J. Bifurc. Chaos 3, 1529 (1993)

17. A.P. Kuznetsov, N.V. Stankevich, L.V. Turukina, Physica D 238, 1203 (2009)

18. G.B. Ermentrout, Simulating, Analyzing, and Animating Dynamical Systems: A Guide to XPPAUT for Researchers and Students (SIAM, Philadelphia, 2002)

19. E.J. Doedel, 'Auto-07P: Continuation and bifurcation software for ordinary differential equations' with major contributions from A.R. Champneys, T.F. Fairgrieve, Yu.A. Kuznetsov, B.E. Oldeman, R.C. Paffenroth, B. Sandstede, X.J. Wang, and C. Zhang; available from http://cmvl.cs.concordia.ca/auto/

20. T. Kurz, W. Lauterborn, Phys. Rev. A 37, 1029 (1988)

Open Access This is an Open Access article distributed under the terms of the Creative Commons Attribution License (http://creativecommons.org/licenses/by/4.0), which permits unrestricted use, distribution, and reproduction in any medium, provided the original work is properly cited. 